\section{Kidney \\ Blood Pressure Research}

\title{
Clinical and Biological Determinants of Kidney Outcomes in a Population-Based Cohort Study
}

\author{
Idris Guessous ${ }^{a, b} \quad$ Belén Ponte ${ }^{a, c}$ Pedro Marques-Vidala Fred Paccaud ${ }^{a}$ \\ Jean-Michel Gaspoz ${ }^{\mathrm{b}}$ Michel Burnier ${ }^{\mathrm{d}}$ Gérard Waeber \\ Peter Vollenweider ${ }^{\mathrm{e}}$ Murielle Bochud ${ }^{\mathrm{a}}$ \\ aCommunity Prevention Unit, Institute of Social and Preventive Medicine, Centre Hospitalier \\ Universitaire Vaudois, Lausanne University Hospital, Lausanne; ' Unit of Population Epidemiology, \\ Division of primary care medicine, Department of Community Medicine, Primary Care and Emergency \\ Medicine; 'Department of Nephrology, Geneva University Hospitals, Geneva; ${ }^{d}$ Department of

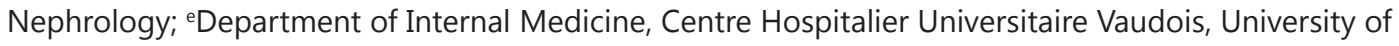 \\ Lausanne, Lausanne, Switzerland
}

\section{Key Words}

Chronic kidney disease $\bullet$ Predictors $\bullet$ Inflammation $•$ Proteinuria

\begin{abstract}
Background/Aims: Prospective studies on factors associated with adverse kidney outcomes in European general populations are scant. Also, few studies consider the potential confounding effect of baseline kidney function. Methods: We used baseline (2003-2006) and 5-year followup data of adults from the general population to evaluate the effect of baseline kidney function and proteinuria on the association of clinical, biological (e.g. uric acid, homocysteine, cytokines), and socioeconomic factors with change in kidney function, rapid decline in kidney function, and incidence of chronic kidney disease (CKD). Estimated glomerular filtration rate (eGFR) and urinary albuminuria-to-creatinine ratio (UACR) were collected. Kidney outcomes were modeled using multivariable regressions. Results: A total of 4,441 subjects were included in the analysis. Among participants without CKD at baseline, $11.4 \%$ presented rapid decline in eGFR and/or incident CKD. After adjustment for baseline eGFR and log UACR, only age (Odds Ratio; 1.25 [95\%CI 1.18-1.33]), diabetes (OR 1.48 [1.03-2.13]), education (OR middle vs. high 1.51 [1.08-2.11]) and log ultrasensitive CRP (OR 1.16 [1.05-1.22]) were associated with rapid decline in eGFR or incident CKD. Baseline log UACR (OR 1.18 [1.06-1.32]) but not eGFR was associated with rapid decline in eGFR and/or incident CKD. Conclusion: In addition to age and diabetes, education and CRP levels are associated with adverse kidney outcomes independently of baseline kidney function.
\end{abstract}




\section{Kidney Blood Pressure Research}

Kidney Blood Press Res 2014;39:74-85

\begin{tabular}{l|l}
\hline DOI: $10.1159 / 000355779$ & (c) 2014 S. Karger AG, Basel
\end{tabular}

Publisned on Ine: June 23,2014

www.karger.com/kbr

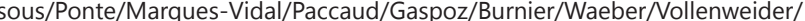
Bochud: Determinants of Kidney Outcomes

\section{Introduction}

Chronic kidney disease (CKD) is usually defined as the persistence for 3 or more months of structural and/or functional abnormalities of the kidney [1]. CKD is the $12^{\text {th }}$ leading cause of death worldwide and the $9^{\text {th }}$ leading cause of death in the USA [2], and is associated with increased risk of ischemic heart disease, stroke, peripheral vascular disease, anemia, bone disease, end-stage renal disease (ESRD), and mortality [3]. Recently, change in kidney function in subjects without CKD has been associated with adverse outcomes, including mortality [4, 5]. For example, rapid kidney decline - defined as an annual decline in estimated glomerular filtration rate (eGFR) greater than $3 \mathrm{~mL} / \mathrm{min} / 1.73 \mathrm{~m}^{2}$ - has been associated with increased risk of cardiovascular events [6].

Clinical (e.g., age, smoking, diabetes, body mass index), biological (e.g., uric acid, HDLcholesterol, triglycerides, homocysteine, $\mathrm{C}$ reactive protein, cytokines), and socioeconomic (e.g., education) factors associated with incident CKD and/or change in eGFR have been reported in prospective studies [7-17]. In addition to these factors, baseline kidney function (defined by eGFR or proteinuria) is independently associated with incident CKD and/or change in eGFR [18-24]. In fact, proteinuria seems to be a better predictor of loss of renal function than eGFR [25]. Still, most prospective studies have been conducted in North America and data on incidence of and factors associated with kidney outcomes in European populations are scant [26].

Further, previous prospective studies have rarely considered the impact of baseline kidney function on the association of clinical, biological, or socioeconomic factors on incident CKD or change in eGFR. Those who did generally found a change in the associations [16, 23 ,27], suggesting that the relationships, if any, might be confounded by baseline kidney function [21].

We used baseline (2003-2006) and 5-year follow-up data of adults from the general population to evaluate the association of clinical, biological, and socioeconomic factors with change in kidney function, rapid decline in kidney function, and incidence of CKD. We then determined the effect of baseline kidney function and proteinuria on these associations.

\section{Subjects and Methods}

\section{Colaus}

We used the data from the CoLaus study. The primary aims of the CoLaus study was to assess the prevalence and determinants of cardiovascular disease in the Caucasian population of Lausanne, Switzerland $[28,29]$. The CoLaus study complied with the Declaration of Helsinki and was approved by the local Institutional Ethics Committee. All participants gave written informed consent. The sampling procedure of the CoLaus study has been described elsewhere [28]. Briefly, the CoLaus study was populationbased and included participants aged 35 to 75 years. The recruitment took place in the city of Lausanne in Switzerland, a town of 117,161 inhabitants, of which 79,420 are of a Swiss nationality. The complete list of the Lausanne inhabitants aged $35-75$ years $(n=56,694$ in 2003) was provided by the population register of the city and served to sample the participants to the study. A simple, non-stratified random sample of 35\% of the overall population was drawn. The following inclusion criteria applied: a) written informed consent; b) aged 35-75 years; c) willingness to take part in the examination and donate blood. Recruitment began in June 2003 and ended in May 2006. The sample of 8,121 subjects who agreed to participate represented $41 \%$ of the initially sampled population. Participants were asked to attend the outpatient clinic at the Centre Hospitalier Universitaire Vaudois (CHUV) in the morning after an overnight fast. Between 2009 and 2012, all CoLaus participants have been invited for a follow-up (CoLaus 2). Among these, 4,679/6,188 (75.6\%) Caucasian subjects participated to the follow-up.

\section{Assessment process and clinical data}

Data were collected by trained field interviewers, using standardized questionnaires. Questionnaires recorded information on demographic data, socioeconomic status, and several lifestyle factors such as 


\section{Kidney Blood Pressure Research}

Kidney Blood Press Res 2014;39:74-85

\begin{tabular}{l|l}
\hline DOI: $10.1159 / 000355779$ & (c) 2014 S. Karger AG, Basel
\end{tabular}

Publisned ontıne: June 23,2014

www.karger.com/kbr

(1)/Ponte/Marques-Vidal/Paccaud/Gaspoz/Burnier/Waeber/Vollenweider/ Bochud: Determinants of Kidney Outcomes

tobacco use and physical activity. A questionnaire, administered during a face-to-face meeting with the recruiter, focused on personal and family history of disease and cardiovascular risk factors. Use of oral contraception and hormonal replacement therapy was self-reported.

Blood pressure (BP) was measured thrice on the left arm after at least 10 minutes rest in the seated position using a clinically validated oscillometric device (Omron® HEM-907, Matsusaka, Japan) [30]. The average of the last two BP readings was used for analyses. Hypertension was defined as mean systolic BP (SBP) $\geq 140 \mathrm{mmHg}$ or mean diastolic BP (DBP) $\geq 90 \mathrm{mmHg}$ or presence of anti-hypertensive medication. Diabetes was defined as a fasting glucose $\geq 7 \mathrm{mmol} / \mathrm{L}$ and/or presence of antidiabetic drug treatment (insulin or oral drugs). In addition, weight, height, and waist and hip circumferences were measured using standardized procedures. Body mass index (BMI) was defined as weight/height².

\section{Biologic data}

Venous blood samples were drawn after an overnight fast. Glucose was measured by Glucose dehydrogenase (Roche Diagnostics, $\mathrm{CH}$; CV 2.1\%-1.0\%), total serum cholesterol, HDL-cholesterol and serum triglycerides were measured by glycerol-3-phosphate oxidase - phenol aminophenazone (GPO-PAP) (Roche Diagnostics, $\mathrm{CH}$; CV 3.6\%-0.5\%). Total serum calcium was measured by O-cresolphtalein (Roche Diagnostics, $\mathrm{CH}$; CV 2.1\%-1.5\%). Albumin-corrected calcium was calculated using the following formula: $\mathrm{Ca}_{c}$ $=$ Serum total calcium $-0.012 \times($ serum albumin $/ 0.9677-39.55)$. Ultrasensitive $\mathrm{C}$ reactive protein (hsCRP) was measured by Immunoassay and latex HS (Agilent 1100 apparatus, CH; CV 4.6\%-1.3\%). Cytokine levels were measured using a multiplexed particle-based flow cytometric assay [31]. Milliplex kits were purchased from Millipore (Zug, Switzerland). The analysis was conducted using a conventional flow cytometer (FC500 MPL, Beckman Coulter, Nyon, Switzerland). Lower detection limits for IL-1 $\beta$, IL-6 and TNF- $\alpha$ were 0.2 pg/ $\mathrm{ml}$. Intra and inter-assay coefficients of variation were $15 \%$ and $16.7 \%$ for IL-1 $\beta, 16.9 \%$ and $16.1 \%$ for IL-6 and $12.5 \%$ and $13.5 \%$ for TNF- $\alpha$, respectively. Serum uric acid and homocysteine were measured by uricasePAP (Roche Diagnostics, CH, CV 1.0\%-0.5\%) and high pressure liquid chromatography following ammonium 7-fluorobenzo-2-oxa-1, 3-diazole-4-sulphonate (SBD-F) derivatisation (Agilent 1100 apparatus, CV 3.1\%$2.9 \%$ ), respectively.

A single spot urine sample was taken in the morning. Creatinine and albumin were measured and urinary albumin-to-creatinine ratio (UACR) calculated to estimate proteinuria. Albumin (serum and urine) was measured by bromocresol green (Roche Diagnostics, $\mathrm{CH}$; CV 2.5\%-0.4\%) and creatinine (serum and urine) by IDMS-traceable Jaffe kinetic compensated method (Roche Diagnostics CH; CV 2.9\%-0.7\%). Albuminuria was defined as UACR of $\geq 30 \mathrm{mg} / \mathrm{g}$. The National Kidney Foundation clinical practice guidelines definition of CKD was used[1]; eGFR $<60 \mathrm{ml} / \mathrm{min} / 1.73 \mathrm{~m}^{2}$. eGFR was calculated using the Chronic Kidney Disease Epidemiology Collaboration (CKD-EPI) equation [32].

\section{Adverse kidney outcomes definition}

Annual change in eGFR was defined as the annualized difference between eGFR at follow-up and eGFR at baseline. Rapid decline in eGFR was defined as an annual loss $>3 \mathrm{~mL} / \mathrm{min} / 1.73 \mathrm{~m}^{2}$, as previously suggested [4]. Incident CKD was defined as an eGFR $<60 \mathrm{~mL} / \mathrm{min} / 1.73 \mathrm{~m}^{2}$ at follow-up among participants with eGFR $\geq 60 \mathrm{~mL} / \mathrm{min} / 1.73 \mathrm{~m}^{2}$ at baseline. Of note, creatinine was measured using the same methods at baseline and at follow-up. Because of the low number of participants with incident CKD at follow-up, rapid decline in eGFR and/or incident CKD outcomes were combined among participants without CKD at baseline. Results for incident CKD outcome only are presented in the supplementary material.

\section{Statistical Analyses}

Statistical analyses were performed using Stata 12.0 software (Stata Corp, College Station, TX, USA). Results were expressed as number of participants (percentage, \%), mean (standard deviation, SD) or median and interquartile range (IQR). Between-group comparisons were conducted using chi-square, Student's, or Wilcoxon ranksum tests. All values of IL-1 $\beta$, IL- 6 and TNF- $\alpha$ below the detection level $(0.2 \mathrm{pg} / \mathrm{ml})$ were substituted with a value $(0.133)$ equivalent to two-thirds of the lower detection limit as recommended by Hornung et al [33]. Annual change in eGFR was modeled as a function of clinical, biological, and socioeconomic factors at baseline using linear regression, and results were expressed as annual difference and 95\% confidence interval (95\%CI). Rapid decline in eGFR, incident CKD, and the combined outcome 


\section{Kidney Blood Pressure Research}

Kidney Blood Press Res 2014;39:74-85

\begin{tabular}{l|l}
\hline DOI: 10.1159/000355779 & (c) 2014 S. Karger AG, Basel
\end{tabular}

Published ontıne: June 23,2014

www.karger.com/kbr

Guessous/Ponte/Marques-Vidal/Paccaud/Gaspoz/Burnier/Waeber/Vollenweider/ Bochud: Determinants of Kidney Outcomes

(rapid decline in eGFR or incident CKD) were modeled separately as a function of clinical, biological, and socioeconomic factors at baseline using unconditional logistic regression, and the results were expressed as odds ratio (OR) and 95\% CI. To determine the impact of kidney function at baseline on these associations, we sequentially adjusted for eGFR at baseline and UACR at baseline. To satisfy regression assumptions, UACR, hsCRP, IL-6, IL-1 $\beta$, and TNF- $\alpha$ were log-transformed.

Variables Specification

Risk factors for kidney function decline were selected from earlier reports and multivariable regression models built using the same method used by O'Seaghdha et al. in the Framingham Heart Study and Atherosclerosis Risk in Communities Study [7]. Variables were sequentially added in a pre-specified order and incorporated using a P value $<0.05$ threshold for entry and retention in the final model.

Candidates for entry in order of inclusion were: age, sex, diabetes, hypertension, HDL- cholesterol, total cholesterol, triglycerides, BMI, smoking status, alcohol consumption, physical activity, education, contraceptive pill, menopause, albumin-corrected calcium, hsCRP, IL-1 $\beta$, IL-6, TNF- $\alpha$, uric acid, and homocysteine. Education was assessed as the highest qualification achieved and categorized as "high" (tertiary education), "middle" (upper secondary education or post-secondary non tertiary education, including vocational education) and "low" (lower secondary education or lower). Baseline eGFR and baseline log UACR were then sequentially added to the models. We only included in the analysis Caucasian subjects for whom all variables of interest for the purpose of this study were available.

\section{Results}

Participant's Characteristics

Out of the 4,679 participants to the Colaus follow-up study, $238(5.1 \%)$ had at least one missing value for all variables of interest, leaving a total of 4,441 participants (94.9\%) for the analysis. The mean (SD) follow-up time was $5.6(0.5)$ years and the median (IQR) 5.5 (IQR 5.4-5.7) years. At baseline, the overall mean age was 52.6 (10.6) years, $53.5 \%$ were women and $4.3 \%$ had CKD. Characteristics by CKD status at baseline are detailed in Table 1 . The groups defined by CKD status statistically differed by all characteristics apart from gender, HDL-cholesterol, education, physical activity, alcohol consumption, and IL-6. Baseline eGFR and UACR were $87.1(13.4) \mathrm{mL} / \mathrm{min} / 1.73 \mathrm{~m}^{2}$ and $12.9(60.5) \mathrm{mg} / \mathrm{g}$ in participants without CKD at baseline and $52.6(7.7) \mathrm{mL} / \mathrm{min} / 1.73 \mathrm{~m}^{2}$ and 27.1 (69.1) $\mathrm{mg} / \mathrm{g}$ in participants with CKD at baseline (both P values $<0.001)$. Overall, 5.7\% (253/4441) of participants presented albuminuria at baseline.

The mean annual eGFR change was $-0.632(1.72) \mathrm{mL} / \mathrm{min} / 1.73 \mathrm{~m}^{2}$ and $0.528(2.54)$ in subjects without and with CKD at baseline, respectively (P value $<0.001$, Table 2 ). Among all subjects, annual decline in eGFR was steeper among participants with albuminuria at baseline than participants without albuminuria at baseline: -0.876 (2.34) vs. -0.566 (1.73), P value $<0.001$. Among participants with CKD at baseline, annual change in eGFR was -0.404 (2.11) and $0.700(2.58)$ in participants with $(29 / 189,15.3 \%)$ and without albuminuria $(160 / 189,84.7 \%)$ at baseline, respectively (P value=0.031). Rapid decline in eGFR tended to be more frequent among participants without CKD at baseline than participants with CKD at baseline $(7.0 \%$ vs. $3.7 \%$, P value $=0.077)$, though not statistically significant. Out of the participants without CKD at baseline, 186 (4.4\%) presented an incident CKD at followup, $485(11.4 \%)$ presented a rapid decline in eGFR or an incident CKD at follow-up, and 71 $(1.7 \%)$ presented both a rapid decline in eGFR and an incident CKD at follow-up.

Tables 3, 4 and 5 report the associations of risk factors with annualized change in eGFR, rapid decline in eGFR and rapid decline in eGFR and/or incident CKD (combined outcome), without adjustment for kidney function at baseline (Table 3), with adjustment for eGFR at baseline (Table 4), and with adjustment for both baseline eGFR and baseline log UACR (Table 5). 


\section{Kidney \\ Blood Pressure Research}

Table 1. Baseline Characteristics of the CoLaus participants, by chronic kidney disease (CKD) status at baseline $(\mathrm{N}=4441)$

\begin{tabular}{|c|c|c|c|}
\hline & $\begin{array}{c}\text { No CKD } \\
\text { eGFR } \geq 60 \mathrm{ml} \mathrm{mL} / \\
\min / 1.73 \mathrm{~m}^{2} \\
\mathrm{~N}=4252(95.7 \%)\end{array}$ & $\begin{array}{c}\text { CKD } \\
\text { eGFR }<60 \mathrm{ml} \\
\mathrm{mL} / \mathrm{min} / 1.73 \mathrm{~m}^{2} \\
\mathrm{~N}=189(4.3 \%)\end{array}$ & $P$ value \\
\hline Age mean (SD) & $52.1(10.3)$ & $64.1(8.9)$ & $<0.001$ \\
\hline Female gender $\mathrm{N}(\%)$ & $2261(53.2)$ & $113(59.8)$ & 0.074 \\
\hline Type 2 diabetes $\mathrm{N}(\%)$ & $226(5.3)$ & $24(12.7)$ & $<0.001$ \\
\hline Hypertension N (\%) & $1359(32.0)$ & $123(65.1)$ & $<0.001$ \\
\hline HDL-cholesterol (mmol/L) mean (SD) & $1.65(0.4)$ & $1.63(0.5)$ & 0.571 \\
\hline Total cholesterol (mmol/L) mean (SD) & $5.57(1.0)$ & $5.87(1.1)$ & $<0.001$ \\
\hline Serum triglycerides (mmol/L) mean (SD) & $1.34(0.98)$ & $1.64(1.29)$ & $<0.001$ \\
\hline 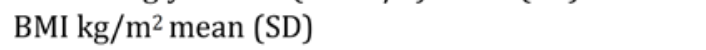 & $25.6(4.4)$ & $26.9(4.4)$ & $<0.001$ \\
\hline Smoking status & & & $<0.001$ \\
\hline Never smokers N (\%) & $1724(40.5)$ & $83(43.9)$ & \\
\hline Former smokers N (\%) & $1421(33.4)$ & $81(42.9)$ & \\
\hline Smokers N (\%) & $1107(26.0)$ & $25(13.2)$ & \\
\hline Current alcohol consumption $\mathrm{N}(\%)$ & $3444(81.0)$ & $153(80.9)$ & 0.983 \\
\hline Physical activity & & & 0.545 \\
\hline Never or don't know $\mathrm{N}(\%)$ & $1409(33.1)$ & $57(30.2)$ & \\
\hline 1x/week N (\%) & $405(9.5)$ & $16(8.5)$ & \\
\hline 2x/week N (\%) & $2438(57.3)$ & $116(61.4)$ & \\
\hline Education level & & & 0.164 \\
\hline High $N(\%)$ & $2319(54.5)$ & $108(57.1)$ & \\
\hline Middle N (\%) & $1067(25.1)$ & $53(28.0)$ & \\
\hline Low $\mathrm{N}(\%)$ & $866(20.4)$ & $28(14.8)$ & \\
\hline Contraceptive pill $\mathrm{N}(\%)$ among women & $201(8.9)$ & $2(1.8)$ & 0.008 \\
\hline Menopause N (\%) among women & $1204(53.2)$ & $99(87.6)$ & $<0.001$ \\
\hline Albumin-corrected calcium (mmol/L) mean (SD) & $2.21(0.09)$ & $2.26(0.09)$ & $<0.001$ \\
\hline hsCRP (mg/L) median (IQR) & $1.2(0.6-2.6)$ & $1.6(0.8-3.1)$ & $<0.001$ \\
\hline IL-6 (pg/mL) median (IQR) & $1.3(0.6-3.1)$ & $1.5(0.8-3.1)$ & 0.0860 \\
\hline IL-1 $\beta(\mathrm{pg} / \mathrm{mL})$ median (IQR) & $0.4(0.1-1.8)$ & $0.2(0.1-1.0)$ & $<0.001$ \\
\hline TNF- $\alpha(\mathrm{pg} / \mathrm{mL})$ median (IQR) & $2.8(1.8-4.4)$ & $3.6(2.3-5.3)$ & $<0.001$ \\
\hline Serum uric acid $(\mu \mathrm{mol} / \mathrm{L})$ mean $(\mathrm{SD})$ & $307.9(81.5)$ & $377.1(96.5)$ & $<0.001$ \\
\hline Serum homocysteine $(\mu \mathrm{mol} / \mathrm{L})$ mean $(\mathrm{SD})$ & $10.0(3.7)$ & $13.4(4.3)$ & $<0.001$ \\
\hline eGFR, mL/min/1.73m² mean(SD) & $87.1(13.4)$ & $52.6(7.7)$ & $<0.001$ \\
\hline Albumin-to-creatinine ratio, $\mathrm{mg} / \mathrm{g}$ mean(SD) & $12.9(60.6)$ & $27.1(69.0)$ & 0.002 \\
\hline
\end{tabular}

Table 2. Kidney outcomes, by chronic kidney disease (CKD) status at baseline $(\mathrm{N}=4441)$

\begin{tabular}{lccc}
\hline & $\begin{array}{c}\text { No CKD; } \\
\text { eGFR } \geq 60 \mathrm{ml}, \\
\mathrm{mL} / \mathrm{min} / 1.73 \mathrm{~m}^{2}, \\
\mathrm{~N}=4252(95.7 \%)\end{array}$ & $\begin{array}{c}\text { CKD; } \\
\text { eGFR }<60 \mathrm{ml}, \\
\mathrm{mL} / \mathrm{min} / 1.73 \mathrm{~m}^{2}, \\
\mathrm{~N}=189(4.3 \%)\end{array}$ & P value \\
\hline Annual change in eGFR, $\mathrm{mL} / \mathrm{min} / 1.73 \mathrm{~m}^{2}$ (SD) & $-0.632(1.72)$ & $0.528(2.54)$ & $<0.001$ \\
Rapid decline in eGFR, N (\%) & $299(7.1)$ & $7(3.7)$ & 0.077 \\
Incident CKD, N (\%) & $186(4.4)$ & $\mathrm{NA}$ & $\mathrm{NA}$ \\
Rapid decline in eGFR or Incident CKD, N (\%) & $485(11.4)$ & $\mathrm{NA}$ & $\mathrm{NA}$ \\
Rapid decline in eGFR and Incident CKD, N (\%) & $71(1.7)$ & $\mathrm{NA}$ & $\mathrm{NA}$ \\
\hline \multicolumn{4}{l}{ CKD, chronic kidney disease; eGFR, estimated glomerular filtration rate; NA: not appropriate } \\
\hline
\end{tabular}




\section{Kidney Blood Pressure Research}

\begin{tabular}{|c|c|}
\hline Kidney Blood Press Res & \\
\hline $\begin{array}{l}\text { DOI: } 10.1159 / 000355779 \\
\text { Publisned online: June } 23,2014\end{array}$ & $\begin{array}{l}\text { (c) } 2014 \text { S. Karger AG, Base } \\
\text { www.karger.com/kbr }\end{array}$ \\
\hline
\end{tabular}

Table 3. Associations of baseline characteristics with kidney outcomes, unadjusted for baseline kindey function

\begin{tabular}{|c|c|c|c|c|c|c|c|c|c|}
\hline \multirow[t]{2}{*}{ Covariates } & \multicolumn{3}{|c|}{$\begin{array}{c}\text { Annual change in eGFR } \\
\left(\mathrm{N}^{*}=4441\right)\end{array}$} & \multicolumn{3}{|c|}{$\begin{array}{l}\text { Rapid loss in eGFR } \\
\qquad\left(\mathrm{N}^{*}=4441\right)\end{array}$} & \multicolumn{3}{|c|}{$\begin{array}{l}\text { Rapid loss in eGFR and/or } \\
\text { incident CKD; }\left(\mathrm{N}^{*}=4252\right)\end{array}$} \\
\hline & $\begin{array}{c}\text { Beta } \\
\text { coefficients }\end{array}$ & $95 \% \mathrm{CI}$ & $P$ value & OR & $95 \% \mathrm{CI}$ & $P$ value & OR & $95 \% \mathrm{CI}$ & P value \\
\hline Age (5-year) & -0.067 & $-0.095,-0.039$ & $<0.001$ & $\cdots$ & $-\cdot$ & $-\cdot$ & 1.23 & $1.17,1.30$ & $<0.001$ \\
\hline Type 2 diabetes & -0.380 & $-0.614,-0.147$ & 0.001 & 2.20 & $1.49,3.27$ & $<0.001$ & 1.60 & $1.12,2.30$ & 0.010 \\
\hline Hypertension & $-\cdot$ & -. & -. & 1.37 & $1.06,1.77$ & 0.014 & -. & -. & -. \\
\hline HDL-cholesterol (mmol/L) & 0.280 & $0.148,0.412$ & $<0.001$ & - & - & - & $-\cdot$ & - & 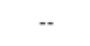 \\
\hline \multicolumn{10}{|l|}{ Smoking status } \\
\hline Never smokers & 0.0 (ref) & & & - & - & -- & $-\cdot$ & $-\cdot$ & $-\cdot$ \\
\hline Former smokers & 0.043 & $-0.079,0.164$ & 0.489 & .. & -. & .. & -. & .. & .. \\
\hline Smokers & -0.132 & $-0.264,-0.001$ & 0.049 & $\cdots$ & 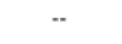 & $\cdots$ & - & $\cdot$ & $\cdots$ \\
\hline \multicolumn{10}{|l|}{ Education level } \\
\hline High & $-\cdot$ & $\cdots$ & $\cdots$ & 1.0 (ref) & & & 1.0 (ref) & & \\
\hline Middle & .- & .. &.- & 1.67 & $1.15,2.43$ & 0.007 & 1.54 & $1.10,2.16$ & 0.011 \\
\hline Low & $-\cdot$ & -. & -. & 1.25 & $0.88,1.78$ & 0.162 & 1.27 & $0.93,1.73$ & 0.134 \\
\hline Contraceptive pill & 0.688 & $0.423,0.952$ & $<0.001$ & -. & - & - & -- & -- & -. \\
\hline Albumin-corrected calcium (mmol/L) & 1.254 & $0.641,1.866$ & $<0.001$ & -. & -. & -. & -. & -. & -. \\
\hline Log hsCRP (mg/L) & -0.073 & $-0.124,-0.022$ & 0.005 & 1.15 & $1.05,1.40$ & 0.015 & 1.18 & $1.07,1.30$ & 0.001 \\
\hline Serum uric acid $(\mu \mathrm{mol} / \mathrm{L})$ & 0.002 & $0.001,0.002$ & $<0.001$ & 0.99 & $0.98,0.99$ & 0.022 & $-\cdot$ & -- & -. \\
\hline Serum homocysteine $(\mu \mathrm{mol} / \mathrm{L})$ & 0.022 & $0.007,0.037$ & 0.003 & -. & -. & .- & -. & .. & .. \\
\hline
\end{tabular}

Table 4. Associations of baseline characteristics with kidney outcomes, further adjusted for estimated glomerular filtration rate (CKD-EPI) at baseline

\begin{tabular}{|c|c|c|c|c|c|c|c|c|c|}
\hline \multirow[t]{2}{*}{ Covariates } & \multicolumn{3}{|c|}{$\begin{array}{c}\text { Annual change in eGFR } \\
\left(\mathrm{N}^{*}=4441\right)\end{array}$} & \multicolumn{3}{|c|}{$\begin{array}{c}\text { Rapid loss in eGFR } \\
\left(\mathrm{N}^{*}=4441\right)\end{array}$} & \multicolumn{3}{|c|}{$\begin{array}{l}\text { Rapid loss in eGFR and/or } \\
\text { incident } C K D\left(N^{*}=4252\right)\end{array}$} \\
\hline & $\begin{array}{c}\text { Beta } \\
\text { coefficients }\end{array}$ & $95 \% \mathrm{CI}$ & $P$ value & OR & $95 \% \mathrm{CI}$ & $P$ value & OR & $95 \% \mathrm{CI}$ & P value \\
\hline Age (5-year) & -0.255 & $-0.285,-0.230$ & $<0.001$ & -. & -. & $\ldots$ & 1.27 & $1.19,1.34$ & $<0.001$ \\
\hline Type 2 diabetes & -0.249 & $-0.462,-0.036$ & 0.022 & 2.23 & $1.50,3.33$ & $<0.001$ & 1.57 & $1.09,2.25$ & 0.014 \\
\hline Hypertension & -. & .. & -- & 1.61 & $1.24,2.09$ & $<0.001$ & -. & -. & -. \\
\hline HDL-cholesterol (mmol/L) & 0.113 & $-0.008,0.233$ & 0.066 & -. & -. & .. & -. & .. & -. \\
\hline Smoking status & & & & & -. & & & & \\
\hline Never smokers & 0.0 (ref) & & & -. & & -. & -. & -. & -. \\
\hline Former smokers & 0.053 & $-0.058,0.164$ & 0.348 & .. & -. & .. & -. & .. & .. \\
\hline Smokers & 0.035 & $-0.086,0.157$ & 0.566 & .. & .. & .. & -. & .. & -. \\
\hline Education level & & & & & -- & & & & \\
\hline High & -. & -. & -. & 1.0 (ref) & & & 1.0 (ref) & & \\
\hline Middle & -. & -. & -. & 1.74 & $1.19,2.53$ & 0.004 & 1.53 & $1.09,2.15$ & 0.013 \\
\hline Low & .. & .. & .. & 1.21 & $0.85,1.72$ & 0.289 & 1.24 & $0.91,1.69$ & 0.171 \\
\hline Contraceptive pill & 0.351 & $0.109,0.593$ & 0.004 & -. & -. & -. & -. & -. & -. \\
\hline Albumin-corrected calcium (mmol/L) & 0.698 & $0.138,1.257$ & 0.014 & -. & -. & -. & -. & -. & -. \\
\hline Log hsCRP (mg/L) & -0.054 & $-0.100,-0.006$ & 0.025 & 1.15 & $1.03,1.28$ & 0.012 & 1.18 & $1.07,1.30$ & 0.001 \\
\hline Serum uric acid $(\mu \mathrm{mol} / \mathrm{L})$ & 0.0004 & $-0.0001,0.001$ & 0.162 & 0.99 & $0.99,1.00$ & 0.326 & -. & -- & -. \\
\hline Serum homocysteine $(\mu \mathrm{mol} / \mathrm{L})$ & -0.013 & $-0.026,0.0006$ & 0.062 & .- & -- & -. & -. & -. & -. \\
\hline eGFR at baseline $\left(\mathrm{mL} / \mathrm{min} / 1.73 \mathrm{~m}^{2}\right)$ & -0.060 & $-0.063,-0.056$ & $<0.001$ & 1.04 & $1.03,1.04$ & $<0.001$ & 1.01 & $0.99,1.01$ & 0.082 \\
\hline
\end{tabular}

\section{Annualized change in eGFR}

Age, diabetes, HDL-cholesterol, smoking status, contraceptive pill, albumin-corrected calcium, log hsCRP, uric acid and homocysteine met criteria for inclusion in the model with annualized change in eGFR. Age, diabetes, current smoking status, and log hsCRP were associated with an annual decline in eGFR, whereas HDL-cholesterol, contraceptive pill, albumin-corrected calcium, uric acid and homocysteine were associated with an annual increase in eGFR (Table 3). After adjustment for baseline eGFR, only age, diabetes, contraceptive pill, albumin-corrected calcium, and hsCRP remained associated with annualized change in eGFR (Table 4). Baseline eGFR was negatively associated with annualized change in eGFR: beta coefficient $(\beta):-0.060$ (95\%CI $-0.063,-0.056)$, P value $<0.001$. After further adjustment for baseline log UACR, only age $[\beta$ per 5 -year increase: $-0.253(95 \% \mathrm{CI}-0.280,-0.224)$, $\mathrm{P}$ value $<0.001]$, contraceptive pill [ $\beta$ : 0.356 (95\%CI 0.114, 0.597), $\mathrm{P}$ value $=0.004]$, albumin- 


\section{Kidney \\ Blood Pressure Research}

\begin{tabular}{|l|l|}
\hline DOI: $10.1159 / 000355779$ & C 2014 S. Karger AG, Basel \\
\hline
\end{tabular}

Publisned oninne: June 23,2014

www.karger.com/kbr

Guessous/Ponte/Marques-Vidal/Paccaud/Gaspoz/Burnier/Waeber/Vollenweider/ Bochud: Determinants of Kidney Outcomes

Table 5. Associations of baseline characterisitcs with kidney outcomes, further adjusted for estimated glomerular filtration rate (CKD-EPI) and log urinary albumin-to-creatinine ratio at baseline

\begin{tabular}{|c|c|c|c|c|c|c|c|c|c|}
\hline \multirow[t]{2}{*}{ Covariates } & \multicolumn{3}{|c|}{$\begin{array}{l}\text { Annual change in eGFR } \\
\left(\mathrm{N}^{*}=4441\right)\end{array}$} & \multicolumn{3}{|c|}{$\begin{array}{l}\text { Rapid loss in eGFR } \\
\left(\mathrm{N}^{*}=4441\right)\end{array}$} & \multicolumn{3}{|c|}{$\begin{array}{c}\text { Rapid loss in eGFR and/or } \\
\text { incident CKD }\left(\mathrm{N}^{*}=4252\right)\end{array}$} \\
\hline & $\begin{array}{c}\text { Beta } \\
\text { coefficients }\end{array}$ & $95 \% \mathrm{CI}$ & P value & OR & $95 \% \mathrm{CI}$ & P value & OR & $95 \% \mathrm{CI}$ & $P$ value \\
\hline Age (5-year) & -0.253 & $-0.280,-0.224$ & $<0.001$ & -. & -. & $-\cdot$ & 1.25 & $1.18,1.33$ & $<0.001$ \\
\hline Type 2 diabetes & -0.193 & $-0.409,0.022$ & 0.079 & 2.02 & $1.33,3.04$ & 0.001 & 1.48 & $1.03,2.13$ & 0.032 \\
\hline Hypertension & -. & -. & -. & 1.52 & $1.17,1.99$ & 0.002 & -. & -- & -. \\
\hline HDL-cholesterol (mmol/L) & 0.120 & $-0.0001,0.241$ & 0.050 & -. & -. & -- & -. & -. & -. \\
\hline Smoking status & & & & & -. & & & -. & \\
\hline Never smokers & 0.0 (ref) & & & -. & &.- & -- & & - \\
\hline Former smokers & 0.051 & $-0.060,0.162$ & 0.366 & -. & -. & $-\cdot$ & -. & -- & -. \\
\hline Smokers & 0.043 & $-0.078,0.164$ & 0.490 & -- & -- & -- & -- & -- & $-\cdot$ \\
\hline Education level & & & & & -. & & & .. & \\
\hline High & -. & -. & -. & 1.0 (ref) & & & 1.0 (ref) & & \\
\hline Middle & -- & -- & - & 1.70 & $1.17,2.48$ & 0.006 & 1.51 & $1.08,2.11$ & 0.016 \\
\hline Low & $-\cdot$ & -- & -- & 1.18 & $0.83,1.68$ & 0.354 & 1.22 & $0.89,1.66$ & 0.214 \\
\hline Contraceptive pill & 0.356 & $0.114,0.597$ & 0.004 & $-\cdot$ & $-\cdot$ & $-\cdot$ & $-\cdot$ & $-\cdot$ & $-\cdot$ \\
\hline Albumin-corrected calcium (mmol/L) & 0.724 & $0.165,1.283$ & 0.011 & $-\cdot$ & $\cdot-$ & $-\cdot$ & -. & $-\cdot$ & -. \\
\hline $\log$ hsCRP $(\mathrm{mg} / \mathrm{L})$ & -0.048 & $-0.095,-0.001$ & 0.043 & 1.14 & $1.02,1.27$ & 0.022 & 1.16 & $1.05,1.28$ & 0.002 \\
\hline Serum uric acid $(\mu \mathrm{mol} / \mathrm{L})$ & 0.0004 & $-0.0001,0.001$ & 0.162 & 0.99 & $0.99,1.00$ & 0.364 &.- & -- & -- \\
\hline Serum homocysteine $(\mu \mathrm{mol} / \mathrm{L})$ & -0.012 & $-0.026,0.0011$ & 0.072 & $-\cdot$ & $-\cdot$ & $-\cdot$ & $-\cdot$ & $-\cdot$ & $-\cdot$ \\
\hline eGFR at baseline $\left(\mathrm{mL} / \mathrm{min} / 1.73 \mathrm{~m}^{2}\right)$ & -0.059 & $-0.063,-0.055$ & $<0.001$ & 1.03 & $1.03,1.04$ & $<0.001$ & 1.00 & $0.99,1.01$ & 0.148 \\
\hline Log UACR & -0.087 & $-0.141,-0.033$ & 0.002 & 1.17 & $1.04,1.32$ & 0.010 & 1.18 & $1.06,1.32$ & 0.002 \\
\hline
\end{tabular}

corrected calcium [ $\beta$ : $0.724(95 \% \mathrm{CI} 0.165,1.283), \mathrm{P}$ value $=0.011]$, and log hsCRP [ $\beta:-0.048$ (95\% CI $-0.095,-0.001), P$ value $=0.043]$ remained associated with annualized change in eGFR (Table 5). Both baseline eGFR and baseline log UACR were negatively associated with annualized change in eGFR.

\section{Rapid decline in eGFR}

Diabetes, hypertension, middle education level, log hsCRP, and uric acid met criteria for inclusion in the model with rapid decline in eGFR. Except uric acid, all variables were positively associated with rapid decline in eGFR (Table 3). After adjustment for baseline eGFR, diabetes [Odds Ratio OR: 2.23 (95\%CI 1.50-3.33), P value <0.001], hypertension [OR: 1.61 (95\%CI 1.24-2.09), P value <0.001], education level (OR middle vs. high: 1.74 (95\%CI 1.19-2.53), $\mathrm{P}$ value=0.004], and log hsCRP [OR: 1.15 (95\%CI 1.03-1.28), $\mathrm{P}$ value $=0.012$ ] remained significantly associated with rapid decline in eGFR whereas uric acid was no longer associated (Table 4). Similar results were observed after further adjustment for baseline log UACR (Table 5). Baseline eGFR and log UACR were positively associated with rapid decline in eGFR.

\section{Combined outcome (rapid decline in eGFR and/or incident CKD)}

Age, diabetes, education level, and log hsCRP met criteria for inclusion for combined outcome (rapid decline in eGFR and/or incident CKD) model. All were positively associated with the combined outcome (Table 3). Similar results were observed after further adjustment for baseline eGFR and further adjustment for log UACR (Tables 4 and 5). After adjustment for both baseline eGFR and log UACR, age [OR per 5-year increase: 1.25 (95\%CI 1.18-1.33), $P$ value $<0.001$ ]; diabetes [OR: 1.48 (95\%CI 1.03-2.13), $\mathrm{P}$ value $=0.032$ ], education level [OR middle vs. high: 1.51 (95\%CI 1.08-2.11), P value=0.016], and log hsCRP [OR: 1.16 (95\%CI 1.05-1.28), $P$ value $=0.002]$ remained associated with the combined outcome (Table 5). Baseline log UACR [OR: 1.18 (95\%CI 1.06-1.32), P value=0.002] but not baseline eGFR was positively associated with the combined outcome.

IL-6, IL-1 $\beta$, and TNF- $\alpha$ were not associated with kidney outcomes in models that included hsCRP. 


\section{Kidney Blood Pressure Research}

Kidney Blood Press Res 2014;39:74-85

DOI: $10.1159 / 000355779$

Published online: June 23,2014

(C) 2014 S. Karger AG, Base

www.karger.com/kbr

Table 6. Associations of baseline characteristics with incident chronic kidney disease (CKD), with and without adjsutement for kidney function at baseline

\begin{tabular}{|c|c|c|c|c|c|c|c|c|c|}
\hline \multirow[t]{2}{*}{ Covariates } & \multicolumn{3}{|c|}{$\begin{array}{l}\text { Incident CKD }\left(\mathrm{N}^{*}=4252\right) \\
\text { No adjustment for kidney } \\
\text { function at baseline }\end{array}$} & \multicolumn{3}{|c|}{$\begin{array}{c}\text { Incident CKD }\left(\mathrm{N}^{*}=4252\right) \\
\text { Adjustment for eGFR at } \\
\text { baseline }\end{array}$} & \multicolumn{3}{|c|}{$\begin{array}{l}\text { Incident } \mathrm{CKD}\left(\mathrm{N}^{*}=4252\right) \\
\text { Adjustment for eGFR and } \\
\text { logUACR at baseline }\end{array}$} \\
\hline & OR & $95 \% \mathrm{CI}$ & P value & OR & $95 \% \mathrm{CI}$ & P value & OR & $95 \% \mathrm{CI}$ & $P$ value \\
\hline Age (5-year) & 1.89 & $1.72,2.08$ & $<0.001$ & 1.56 & $1.41,1.73$ & $<0.001$ & 1.52 & $1.37,1.69$ & $<0.001$ \\
\hline Type 2 diabetes & 1.56 & $1.09,2.43$ & 0.006 & 2.24 & $1.37,3.68$ & 0.001 & 1.94 & $1.17,3.22$ & 0.010 \\
\hline Log hsCRP (mg/L) & 1.31 & $1.12,1.53$ & 0.001 & 1.40 & $1.19,1.65$ & $<0.001$ & 1.37 & $1.16,1.62$ & $<0.001$ \\
\hline Serum uric acid $(\mu \mathrm{mol} / \mathrm{L})$ & 1.01 & $1.01,1.02$ & 0.003 & 1.00 & $0.99,1.02$ & 0.347 & 1.00 & $0.99,1.01$ & 0.512 \\
\hline eGFR at baseline $\left(\mathrm{mL} / \mathrm{min} / 1.73 \mathrm{~m}^{2}\right)$ & NA & NA & NA & 0.90 & $0.88,0.92$ & $<0.001$ & 0.90 & $0.88,0.92$ & $<0.001$ \\
\hline Log UACR & NA & NA & NA & NA & NA & NA & 1.32 & $1.13,1.55$ & 0.001 \\
\hline
\end{tabular}

Supplementary analysis: incident CKD

Age, diabetes, log hsCRP, and uric acid met criteria for inclusion for incident CKD model. All were positively associated with incident CKD (Table 6). But only age, diabetes, log hsCRP remained associated with incident CKD after further adjustment for kidney function at baseline (Table 6). Baseline eGFR and log UACR were respectively negatively [OR: 0.90 (95\%CI 0.88-0.92), P value $<0.001$ ] and positively [OR: 1.32 (95\%CI 1.13-1.55), P value $=0.001]$ associated with incident CKD.

\section{Discussion}

In this prospective population-based study on adults with predominantly normal baseline kidney function, we identified risk factors of change in eGFR, rapid decline in eGFR and incident CKD that are independent of baseline kidney function. We found that baseline proteinuria increased the risk of decline in eGFR, rapid decline in eGFR, and incident CKD.

While several risk factors of kidney function decline have been reported in previous prospective population-based studies [7, 8, 11-14, 17], most studies did not take into account kidney function at baseline. Homocysteine levels, for example, have been associated with traditional cardiovascular disease (CVD) risk factors [34], but are also associated with GFR and proteinuria. An analysis from the Modification of Diet in Renal Disease (MDRD) Study suggested that prior studies demonstrating an association between homocysteine and CVD risk may have inadequately adjusted for the confounding effects of kidney function [16].

Proteinuria is one of the strongest predictors of CKD and ESRD [24, 35, 36]. Proteinuria also predicts incident hypertension and diabetes $[37,38]$. Not taking into account proteinuria at baseline may therefore lead to confounded associations. Not taking into account baseline GFR may also lead to biased associations because regression to the mean cannot be excluded $[25,39]$. We therefore used predictive models with and without baseline kidney function and found that associations with kidney outcomes were indeed dependent on baseline kidney function. After adjustment for both baseline eGFR and baseline log UACR, smoking status, uric acid and homocysteine levels were no longer associated with annual change in eGFR, and uric acid level was no longer associated with rapid decline in eGFR. This suggests that some prior associations between these factors and kidney outcomes may indeed have been inadequately adjusted for the confounding effects of baseline kidney function.

Our analysis showed that age, diabetes, and log hsCRP were risk factors for adverse kidney outcomes independently of baseline kidney function, a finding in line with previous reports $[7,8,39]$. Age and diabetes per se have been associated with incident CKD in studies that controlled for baseline kidney function $[7,8]$. Data on the independent association of CRP or hsCRP with kidney outcomes are more limited. In a secondary analysis of the MDRD study, 


\section{Kidney Blood Pressure Research}

Kidney Blood Press Res 2014;39:74-85

\begin{tabular}{l|l}
\hline DOI: 10.1159/000355779 & (C) 2014 S. Karger AG, Basel
\end{tabular}

Published onlıne: June 23,2014

www.karger.com/kbr

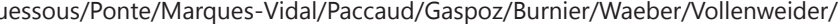
Bochud: Determinants of Kidney Outcomes

CRP level was not associated with rate of decline in kidney function after adjustment for proteinuria [14]. Noteworthly, mean follow-up was only 2.2 years, all participants presented CKD at baseline, and CRP instead of hsCRP was measured. Shankar et al. found no association between hsCRP and long term risk of developing CKD, but their multivariable models did not adjust for baseline eGFR or proteinuria [17]. In the Cardiovascular Health Study (CHS), a prospective study of elderly community-dwelling adults, CRP was positively associated with a decline in eGFR and with decline of eGFR $\geq 3 \mathrm{~mL} / \mathrm{min} / 1.73 \mathrm{~m}^{2} /$ year (rapid decline) after adjustment for baseline creatinine [39]. Our results further suggest that hsCRP, a marker of inflammation, may be an independent predictor of kidney function decrease in adults with predominantly normal kidney function. Several mechanisms relating inflammation and progression of kidney failure have been proposed [14]. For example, inflammation is known from in vivo and in vitro data to play an important role in mediating glomerulosclerosis [40]. Inflammation has been mainly studied in CKD population [41], but rarely in population with predominantly normal kidney function $[17,42]$. Our results suggest that hsCRP level could be considered as an additional independent predictor to be included in the CKD risk score that has been recently proposed for the general population [7]. Of note, cytokines such as IL6 , IL-1 $\beta$, and TNF- $\alpha$ were not independently associated with adverse kidney outcomes when hsCRP was included in multivariable models.

In addition to clinical and biological factors, participants with middle education level presented a greater annual decline in eGFR and were at greater odds of rapid loss in eGFR or incident CKD than participants with high education. Similar associations were found for participants with low education, though not statistically significant. This could be, in part, explain by a lack of power due to the smaller number of participants included in the low education category compared to the other two education categories. Our results are in line with previous studies that have examined the role of education in CKD [11, 43], and also suggest that this association is independent of baseline kidney function. Although the pathophysiological pathways linking education and development of CKD are not fully understood, differences in health care access might be involved [43].

Contrary to baseline eGFR, proteinuria was a strong independent predictor of incident CKD, or CKD in combination with rapid decline in eGFR. We found that annual decline in eGFR was steeper among participants with albuminuria at baseline than participants without albuminuria at baseline. This is consistent with a previous analysis based on 8,592 patients and 24-hour urine collection, which showed that eGFR of individuals with impaired eGFR but without albuminuria stay nearly constant, whereas eGFR deteriorates in individuals with albuminuria [25]. Our findings further suggest that albuminuria screening is a better strategy than eGFR screening to identify individuals who are at risk for accelerated GFR loss.

Of note, we found that the associations of baseline eGFR with rapid decline in eGFR and with combined outcome were different. This difference might be explained, at least in part, by the fact that although the number of participants with incident CKD at follow-up was low, the association of baseline eGFR with incident CKD was negative and significant. Adjusting for the same covariates as the model for the combined outcome (i.e., model adjusted for age, diabetes, education, log hsCRP, and log UACR, see Table 5), baseline eGFR was indeed strongly negatively associated with incident CKD [OR: 0.90 (95\% CI 0.88-0.92), $P$ value $<0.001]$ while baseline eGFR was not associated with the combined outcome as reported in the corresponding Table. The strong negative association of baseline eGFR with incident CKD is however blunted by the positive association we found of baseline eGFR with rapid decline in eGFR explaining the difference observed in the associations of baseline eGFR with rapid decline in eGFR and with combined outcome.

\section{Strengths and limitations}

These results should be interpreted in the light of the study's strengths and limitations. Among the strengths, this is a large prospective study assessing the effect of clinical, biological, and socioeconomic factors on kidney function in a general adult population. Information 


\section{Kidney \\ Blood Pressure Research}

Kidney Blood Press Res 2014;39:74-85

\begin{tabular}{l|l}
\hline DOI: $10.1159 / 000355779$ & C 2014 S. Karger AG, Basel \\
Publisnea ontIne: June 23,2014 & www.karger.com/kbr
\end{tabular}

www.karger.com/kbr

(1)/Marques-Vidal/Paccaud/Gaspoz/Burnier/Waeber/Vollenweider/ Bochud: Determinants of Kidney Outcomes

on major known potential confounders was available. However, information may still be incomplete and residual confounding cannot be excluded. For example, education level was used as a proxy of socioeconomic status, but information on other socioeconomic attributes such as income was not available. GFR was estimated using the CKD-Epi equation and more accurate measurements (including inulin clearance, iothalamate clearance, cystatin C) exist. Still, creatinine is generally used to estimate GFR in clinical practice. Baseline and follow-up serum and urinary creatinine and albumin were measured on a single occasion. Although practice guidelines recommend their use, both creatinine and albumin in serum and urine can show important intraindividual variability [44]. We used UACR based on a single spot to define albuminuria; correlation between UACR and 24-hour collection is high, yet 24-hour collection remains preferable [44]. Finally, our study included only Caucasian participants and our findings may not be generalizable to non-Caucasian populations.

\section{Conclusion}

This prospective population-based study identified risk factors for kidney function deterioration that are independent of baseline kidney function and proteinuria. Age, diabetes, education level, hsCRP, and proteinuria were significantly and independently associated with rapid decline in eGFR and/or incident CKD.

\section{Disclosure Statement}

None declared.

\section{Acknowledgments}

We are grateful to the participants of the CoLaus study and to the investigators, in particular Prof Martin Preisig. The CoLaus study was supported by research grants from GlaxoSmithKline, the Faculty of Biology and Medicine of Lausanne, Switzerland, and the Swiss National Science Foundation (grant no: 33CSCO-122661 and 33CSCO-139468). The funders had no role in study design, data collection and analysis, decision to publish, or preparation of the manuscript.

\section{References}

1 National Kidney Foundation: K/DOQI clinical practice guidelines for chronic kidney disease: evaluation, classification, and stratification. Am J Kidney Dis 2002;39:S1-266.

-2 Schoolwerth AC, Engelgau MM, Hostetter TH, Rufo KH, Chianchiano D, McClellan WM, Warnock DG, Vinicor F: Chronic kidney disease: a public health problem that needs a public health action plan. Prev Chronic Dis 2006;3:A57.

3 Schiffrin EL, Lipman ML, Mann JF: Chronic kidney disease: effects on the cardiovascular system. Circulation 2007;116:85-97.

4 Rifkin DE, Shlipak MG, Katz R, Fried LF, Siscovick D, Chonchol M, Newman AB, Sarnak MJ: Rapid kidney function decline and mortality risk in older adults. Arch Intern Med 2008;168:2212-2218.

-5 Matsushita K, van der Velde M, Astor BC, Woodward M, Levey AS, de Jong PE, Coresh J, Gansevoort RT: Association of estimated glomerular filtration rate and albuminuria with all-cause and cardiovascular mortality in general population cohorts: a collaborative meta-analysis. Lancet 2010;375:2073-2081. 


\section{Kidney \\ Blood Pressure Research}

Kidney Blood Press Res 2014;39:74-85

\begin{tabular}{l|l}
\hline DOI: 10.1159/000355779 & (C) 2014 S. Karger AG, Basel
\end{tabular}

Publisned oninne: June 23,2014

www.karger.com/kbr

6 Shlipak MG, Katz R, Kestenbaum B, Siscovick D, Fried L, Newman A, Rifkin D, Sarnak MJ: Rapid decline of kidney function increases cardiovascular risk in the elderly. J Am Soc Nephrol 2009;20:2625-2630.

-7 O'Seaghdha CM, Lyass A, Massaro JM, Meigs JB, Coresh J, D'Agostino RB, Sr., Astor BC, Fox CS: A risk score for chronic kidney disease in the general population. Am J Med 2012;125:270-277.

-8 Fox CS, Larson MG, Leip EP, Culleton B, Wilson PW, Levy D: Predictors of new-onset kidney disease in a community-based population. JAMA 2004;291:844-850.

-9 de Boer IH, Katz R, Fried LF, Ix JH, Luchsinger J, Sarnak MJ, Shlipak MG, Siscovick DS, Kestenbaum B: Obesity and change in estimated GFR among older adults. Am J Kidney Dis 2009;54:1043-1051.

$\checkmark 10$ Chia YC, Ching SM: Hypertension and the development of new onset chronic kidney disease over a 10 year period: a retrospective cohort study in a primary care setting in Malaysia. BMC Nephrol 2012;13:173.

11 Al-Qaoud TM, Nitsch D, Wells J, Witte DR, Brunner EJ: Socioeconomic status and reduced kidney function in the Whitehall II Study: role of obesity and metabolic syndrome. Am J Kidney Dis 2011;58:389-397.

12 Zhang L, Wang F, Wang X, Liu L, Wang H: The association between plasma uric acid and renal function decline in a Chinese population-based cohort. Nephrol Dial Transplant 2012;27:1836-1839.

13 Tozawa M, Iseki K, Iseki C, Oshiro S, Ikemiya Y, Takishita S: Triglyceride, but not total cholesterol or low-density lipoprotein cholesterol levels, predict development of proteinuria. Kidney Int 2002;62:1743-1749.

14 Sarnak MJ, Poindexter A, Wang SR, Beck GJ, Kusek JW, Marcovina SM, Greene T, Levey AS: Serum C-reactive protein and leptin as predictors of kidney disease progression in the Modification of Diet in Renal Disease Study. Kidney Int 2002;62:2208-2215.

15 Peters AM, Ciapryna MB, Bowles PF, Glass DM: Obesity does not accelerate the decline in glomerular filtration rate associated with advancing age. Int J Obes (Lond) 2009;33:379-381.

16 Menon V, Sarnak MJ, Greene T, Wang X, Pereira AA, Beck GJ, Kusek JW, Selhub J, Collins AJ, Levey AS, Shlipak MG: Relationship between homocysteine and mortality in chronic kidney disease. Circulation 2006;113:1572-1577.

17 Shankar A, Sun L, Klein BE, Lee KE, Muntner P, Nieto FJ, Tsai MY, Cruickshanks KJ, Schubert CR, Brazy PC, Coresh J, Klein R: Markers of inflammation predict the long-term risk of developing chronic kidney disease: a population-based cohort study. Kidney Int 2011;80:1231-1238.

18 Imai E, Horio M, Yamagata K, Iseki K, Hara S, Ura N, Kiyohara Y, Makino H, Hishida A, Matsuo S: Slower decline of glomerular filtration rate in the Japanese general population: a longitudinal 10year follow-up study. Hypertens Res 2008;31:433-441.

19 Li L, Astor BC, Lewis J, Hu B, Appel LJ, Lipkowitz MS, Toto RD, Wang X, Wright JT, Jr., Greene TH: Longitudinal progression trajectory of GFR among patients with CKD. Am J Kidney Dis 2012;59:504512.

20 Vidt DG, Ridker PM, Monyak JT, Schreiber MJ, Cressman MD: Longitudinal assessment of estimated glomerular filtration rate in apparently healthy adults: a post hoc analysis from the JUPITER study (justification for the use of statins in prevention: an intervention trial evaluating rosuvastatin). Clin Ther 2011;33:717-725.

-21 Wang F, Zhang L, Zuo L, Liu L, Wang H: Mortality and renal function decline among a communitybased Chinese population with normal or mildly impaired renal function. Nephrol Dial Transplant 2011;26:2847-2852.

$\longrightarrow 22$ Verhave JC, Gansevoort RT, Hillege HL, Bakker SJ, De Zeeuw D, de Jong PE, PREVEND Study Group: An elevated urinary albumin excretion predicts de novo development of renal function impairment in the general population. Kidney Int Suppl 2004;92:S18-S21.

23 Halbesma N, Jansen DF, Heymans MW, Stolk RP, de Jong PE, Gansevoort RT: Development and validation of a general population renal risk score. Clin J Am Soc Nephrol 2011;6:1731-1738.

24 Turin TC, James M, Ravani P, Tonelli M, Manns BJ, Quinn R, Jun M, Klarenbach S, Hemmelgarn BR: Proteinuria and rate of change in kidney function in a community-based population. J Am Soc Nephrol 2013;24:1661-1667. 


\section{Kidney \\ Blood Pressure Research}

Kidney Blood Press Res 2014;39:74-85

\begin{tabular}{l|l}
\hline DOI: $10.1159 / 000355779$ & (c) 2014 S. Karger AG, Basel
\end{tabular}

Publisned ontıne: June 23,2014

www.karger.com/kbr

Guessous/Ponte/Marques-Vidal/Paccaud/Gaspoz/Burnier/Waeber/Vollenweider/ Bochud: Determinants of Kidney Outcomes

-25 Halbesma N, Kuiken DS, Brantsma AH, Bakker SJ, Wetzels JF, De Zeeuw D, De Jong PE, Gansevoort RT: Macroalbuminuria is a better risk marker than low estimated GFR to identify individuals at risk for accelerated GFR loss in population screening. J Am Soc Nephrol 2006;17:2582-2590.

-26 Lameire N, Jager K, Van Biesen W, de Bacquer D, Vanholder R: Chronic kidney disease: a European perspective. Kidney Int Suppl 2005;99:S30-S38.

-27 Sun X, Chen Y, Chen X, Wang J, Xi C, Lin S, Liu X: Change of glomerular filtration rate in healthy adults with aging. Nephrology (Carlton) 2009;14:506-513.

-28 Firmann M, Mayor V, Vidal PM, Bochud M, Pecoud A, Hayoz D, Paccaud F, Preisig M, Song KS, Yuan X, Danoff TM, Stirnadel HA, Waterworth D, Mooser V, Waeber G, Vollenweider P: The CoLaus study: a population-based study to investigate the epidemiology and genetic determinants of cardiovascular risk factors and metabolic syndrome. BMC Cardiovasc Disord 2008;8:6.

29 Ponte B, Pruijm M, Marques-Vidal P, Martin PY, Burnier M, Paccaud F, Waeber G, Vollenweider P, Bochud M: Determinants and burden of chronic kidney disease in the population-based CoLaus study: a cross-sectional analysis. Nephrol Dial Transplant 2013;28:2329-2339.

-30 El Assaad MA, Topouchian JA, Darne BM, Asmar RG: Validation of the Omron HEM-907 device for blood pressure measurement. Blood Press Monit 2002;7:237-241.

-31 Vignali DA: Multiplexed particle-based flow cytometric assays. J Immunol Methods 2000;243:243255.

32 Levey AS, Stevens LA, Schmid CH, Zhang YL, Castro AF, 3rd, Feldman HI, Kusek JW, Eggers P, Van Lente F, Greene T, Coresh J: A new equation to estimate glomerular filtration rate. Ann Intern Med 2009;150:604-612.

-33 Hornung RW, Reed LR: Estimation of average concentration in the presence of nondetectable values. Appl Occup Environ Hyg 1990;5:46-51.

-34 Ueland PM, Refsum H, Beresford SA, Vollset SE: The controversy over homocysteine and cardiovascular risk. Am J Clin Nutr 2000;72:324-332.

-35 Yamagata K, Ishida K, Sairenchi T, Takahashi H, Ohba S, Shiigai T, Narita M, Koyama A: Risk factors for chronic kidney disease in a community-based population: a 10-year follow-up study. Kidney Int 2007;71:159-166.

-36 Iseki K, Ikemiya Y, Iseki C, Takishita S: Proteinuria and the risk of developing end-stage renal disease. Kidney Int 2003;63:1468-1474.

-37 Brantsma AH, Bakker SJ, de Zeeuw D, de Jong PE, Gansevoort RT: Urinary albumin excretion as a predictor of the development of hypertension in the general population. J Am Soc Nephrol 2006;17:331-335.

-38 Brantsma AH, Bakker SJ, Hillege HL, de Zeeuw D, de Jong PE, Gansevoort RT: Urinary albumin excretion and its relation with C-reactive protein and the metabolic syndrome in the prediction of type 2 diabetes. Diabetes Care 2005;28:2525-2530.

-39 Fried L, Solomon C, Shlipak M, Seliger S, Stehman-Breen C, Bleyer AJ, Chaves P, Furberg C, Kuller L, Newman A: Inflammatory and prothrombotic markers and the progression of renal disease in elderly individuals. J Am Soc Nephrol 2004;15:3184-3191.

$\checkmark 40$ Nakahara C, Kanemoto K, Saito N, Oyake Y, Kamoda T, Nagata M, Matsui A: C-reactive protein frequently localizes in the kidney in glomerular diseases. Clin Nephrol 2001;55:365-370.

41 Pecoits-Filho R, Heimburger O, Barany P, Suliman M, Fehrman-Ekholm I, Lindholm B, Stenvinkel P: Associations between circulating inflammatory markers and residual renal function in CRF patients. Am J Kidney Dis 2003;41:1212-1218.

42 Pruijm M, Ponte B, Vollenweider P, Mooser V, Paccaud F, Waeber G, Marques-Vidal P, Burnier M, Bochud M: Not all inflammatory markers are linked to kidney function: results from a populationbased study. Am J Nephrol 2012;35:288-294.

43 Bruce MA, Beech BM, Crook ED, Sims M, Wyatt SB, Flessner MF, Taylor HA, Williams DR, Akylbekova EL, Ikizler TA: Association of socioeconomic status and CKD among African Americans: the Jackson Heart Study. Am J Kidney Dis 2010;55:1001-1008.

-44 Vu F, Bischoff T, Wolff H, Guessous I, Dory E, Dubois-Arber F, Stringhini S, Bodenmann P: [Social medicine: does it still make sense in 2013?]. Rev Med Suisse 2013;9:2232, 2234-2239. 